Int. J. Morphol.,

32(2):599-607, 2014

\title{
Análisis Crítico de la Literatura Biomédica
}

\author{
Critical Analysis of Biomedical Literature
}

Carlos Manterola ${ }^{*, * * * * * *} \&$ Tamara Otzen ${ }^{* * *, * * * * *}$

MANTEROLA, C. \& OTZEN, T. Análisis crítico de la literatura biomédica. Int. J. Morphol., 32(2):599-607, 2014.

RESUMEN: Ante el gran número de publicaciones existentes y las que día a día incrementan el volumen total, generando un verdadero "bombardeo de información científica"; resulta un verdadero problema el escoger un estudio que responda a las inquietudes clínicas con un buen nivel evidencia. Para ello, se requiere seguir una metodología científica; definiendo y acotando de forma precisa el problema, para luego transformarlo en una pregunta contestable; aplicando posteriormente una estrategia de búsqueda en una fuente de información apropiada; para finalmente, analizar la información de forma crítica y resumir la evidencia encontrada. Para analizar la evidencia se han desarrollado guías de lectura o guías de usuario; de las que existen diversos formatos, pero que en términos generales dan las pautas necesarias para analizar de forma crítica los distintos tipos de artículos biomédicos según sea el escenario clínico al cual pertenezca. Estas herramientas evalúan tres aspectos fundamentales de un estudio: la validez interna, el impacto y la validez externa. Los aspectos antes mencionados se evalúan de forma práctica a través de ejemplo, aplicando una guía de usuario para artículos referentes a tratamiento a un estudio publicado en una prestigiosa revista científica.

PALABRAS CLAVE: Análisis crítico de la literatura; Lectura crítica; Guías de usuario; Validez interna; Validez externa.

\section{INTRODUCTION}

¿Por qué leemos literatura médica? La respuesta está orientada sin duda al hecho que debemos mantenernos informados de los vertiginosos avances científicos que se producen en el ámbito de la medicina y a la necesidad de información que precisamos para resolver problemas clínicos en la atención de nuestros pacientes, tanto en los clásicos ámbitos del diagnóstico y diagnóstico diferencial, tratamiento y pronóstico; como en otros de desarrollo más reciente como los estudios económicos, calidad de vida relacionada a salud (CVRS), etc.

Para lograr esta meta, debemos revisar un gran número de revistas de las que seleccionamos artículos que concitan nuestro interés y que pretendemos leer y evaluar en detalle con posterioridad. Cualquiera sea la razón para leer un artículo científico, debemos ser capaces de leer y evaluar en forma rigurosa el estudio, con el fin de tomar una decisión informada en relación al problema clínico que nos ocupa. Por otra parte, leer en forma científica, nos permite además practicar eventualmente revisiones sistemáticas de la literatura, mediante las cuales se puede analizar y sintetizar la evidencia disponible respecto de una materia particular.
Esta iniciativa surge a principios de la década de los ochenta en el Departamento de Epidemiología Clínica y Bioestadística de la Universidad de McMaster, lugar donde se diseñaron las primeras guías de lectura; las que bajo el apelativo de "Readers' guides", se comenzaron a utilizar para analizar críticamente los artículos referentes a diagnóstico, pronóstico, etiología y tratamiento.

Posteriormente en 1992, se publicaron las modificaciones a las guías de lectura; las que se basaron en la necesidad de dar un enfoque más práctico al análisis de la literatura biomédica. Es decir, que éstas se orientaran a resolver los problemas de la práctica clínica cotidiana. Se trabajó entonces en la perspectiva de la "práctica médica o medicina basada en la evidencia", lo que involucra la habilidad de acceder a la validez e importancia de la evidencia antes de aplicarla a los problemas clínicos del día a día (Manterola, 2009), lo que originó que el concepto de "Reader's Guides" se sustituyese por el de "Users' Guides" (Evidence-Based Medicine Working Group, 1992).

No obstante ello, ante la gran cantidad de artículos

* Departamento de Cirugía y Traumatología, Universidad de La Frontera, Temuco, Chile.

** Centro de Investigación Biomédica, Universidad Autónoma de Chile, Temuco, Chile.

*** Programa de Doctorado en Ciencias Médicas, Universidad de La Frontera, Temuco, Chile.

***** Escuela de Psicología, Universidad Autónoma de Chile, Temuco, Chile.

Parcialmente financiado por Proyecto DIUFRO DI12-0037 
Fuentes

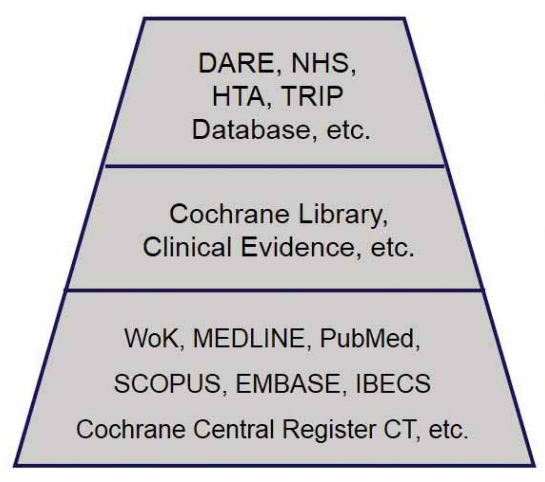

Tipos de estudios
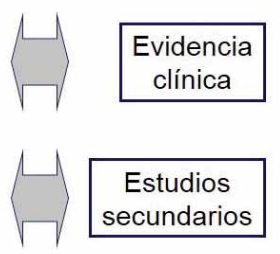

Estudios primarios

Fig. 1. Ejemplos de fuentes de información disponibles y tipos de estudios que se pueden encontrar en cada una de ellas.

publicados, el problema de cómo elegir él o los estudio (s) que aporte (n) repuestas a mis inquietudes clínicas con un buen nivel evidencia es cada vez más frecuente. Por ello, se requiere aplicar una metodología científica; definiendo y acotando de forma precisa el problema, para luego transformarlo en una pregunta contestable que permita obtener una respuesta; aplicando posteriormente una estrategia de búsqueda en una fuente de información apropiada, sea esta de estudios primarios, secundarios o de evidencia clínica (Fig. 1); para finalmente, analizar la información y resumir la evidencia encontrada (Manterola, 2001, Manterola et al., 2006a) (Fig. 2).

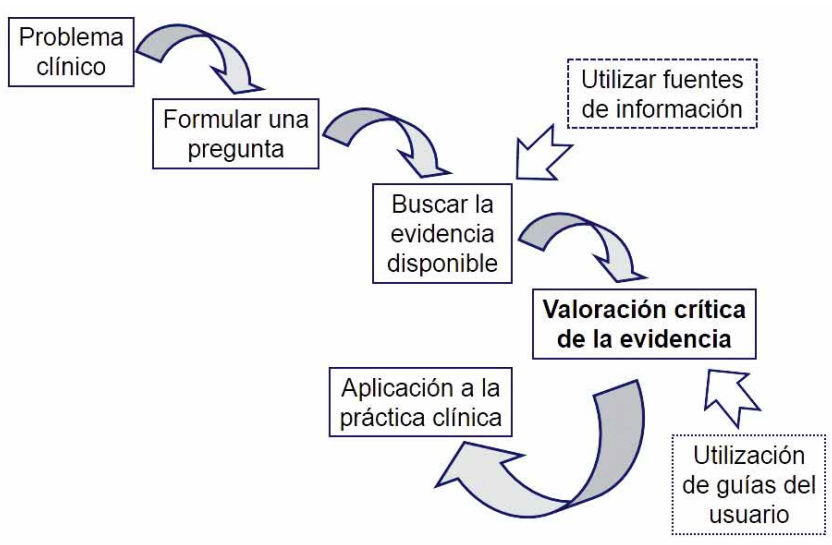

Fig. 2. Diagrama del proceso conducente al análisis crítico de un artículo biomédico.

En este manuscrito, se expondrá de forma sucinta y esquemática algunas características propias del proceso conducente a la lectura crítica de un artículo científico biomédico y su aplicación práctica; para finalizar con el desarrollo de un ejemplo a través de la utilización de una guía de usuario.

\section{¿Cómo analizar la evidencia?}

Para dar respuesta a esta pregunta, se han desarrollado las denominadas guías de lectura; de las que existen diversos formatos, pero que en términos generales dan las pautas necesarias para analizar de forma crítica artículos primarios referentes a tratamiento y prevención, etiología y daño, pronóstico e historia natural, y diagnóstico; y para valorar artículos integradores, secundarios y de sinopsis como son las revisiones sistemáticas de la literatura, los estudios económicos, los análisis de decisiones, las guías de práctica clínica y los estudios de CVRS (Fig. 3).

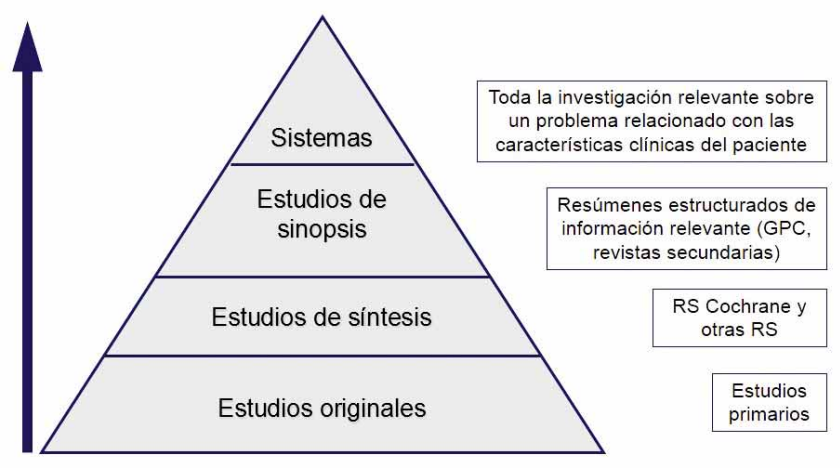

Fig. 3. Tipos de estudios disponibles en relación a su origen y capacidad de síntesis.

Y, ¿cuáles son los pasos a seguir? En primer lugar, se ha de preguntar cómo puedo seleccionar un trabajo útil. Para ello, hemos de considerar las siguientes interrogantes respecto del artículo en evaluación: ¿Sirve para responder a mi pregunta?, ¿otorga datos para buscar una respuesta a mi pregunta?, ¿menciona qué métodos usar para responder mi pregunta?, ¿informa sobre la respuesta que se dio a mi pregunta en otras condiciones?

Si nos convencemos de que el artículo vale la pena leerlo, pasamos a la etapa de la evaluación crítica de este. Para ello, se han de evaluar tres aspectos fundamentales: la validez interna, el impacto y la validez externa (Fig. 4).

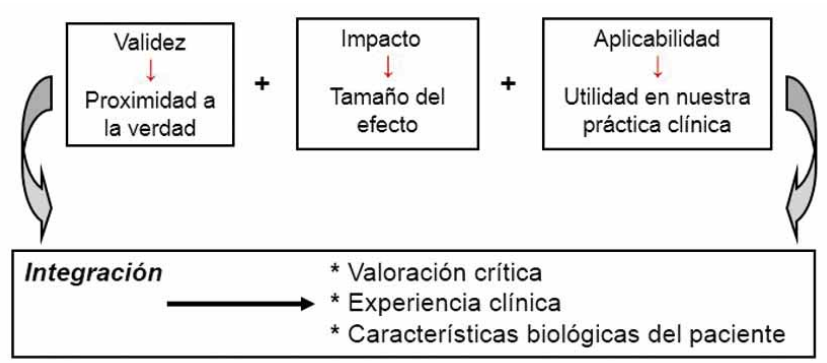

Fig. 4. Etapas de una evaluación crítica de un artículo y sus significados. 
A

\section{A.- ¿SON VÁLIDOS LOS RESULTADOS DEL ESTUDIO?}

Preguntas de "eliminación"

GUÍA

\begin{tabular}{|c|c|}
\hline $\begin{array}{l}\text { 1. ¿Se orienta el articulo a una pregunta claramente } \\
\text { definida? } \\
\text { - Si } \\
\text { - No } \\
\text { - No se puede decir }\end{array}$ & $\begin{array}{l}\text { PISTAS } \\
\text { Una pregunta debe definirse en términos de: } \\
\text { - La población en estudio } \\
\text { - La intervención realizada } \\
\text { - Los resultados considerados }\end{array}$ \\
\hline $\begin{array}{l}\text { 2. ¿Fue aleatoria la asignación de los pacientes al } \\
\text { tratamiento? } \\
\text { - Si } \\
\text { - No } \\
\text { - No se puede decir }\end{array}$ & $\begin{array}{l}\text { PISTA } \\
\text { • ¿Se mantuvo oculta la secuencia de laleatorización? }\end{array}$ \\
\hline $\begin{array}{l}\text { 3. ¿Fueron considerados hasta el final del estudio } \\
\text { todos los pacientes? } \\
\text { - Si } \\
\text { - No } \\
\text { - No se puede decir }\end{array}$ & $\begin{array}{l}\text { PISTAS } \\
\text { - ¿El seguimiento fue completo? } \\
\text { ¿Los pacientes fueron analizados en los grupos a los } \\
\text { que fueron aleatorizados? }\end{array}$ \\
\hline
\end{tabular}

B

Preguntas de "detalle"

GUIA

COMENTARIOS

\begin{tabular}{|c|c|}
\hline $\begin{array}{l}\text { 1. ¿Estuvieron los pacientes, el personal de salud y } \\
\text { el personal involucrado en el estudio enmascarados } \\
\text { respecto del tratamiento? } \\
-\mathrm{Si} \\
\text { - No } \\
\text { - No se puede decir }\end{array}$ & $\begin{array}{l}\text { PISTAS: } \\
\text { - Los pacientes } \\
\text { - El personal de salud que aplica las terapias } \\
\text { - El personal del estudio que realiza las mediciones }\end{array}$ \\
\hline $\begin{array}{l}\text { 2. ¿Los grupos en estudio ¿eran similares al inicio } \\
\text { del estudio? } \\
\text { - Si } \\
\text { - No } \\
\text { - No se puede decir }\end{array}$ & $\begin{array}{l}\text { PISTA } \\
\text { - Verificar otros factores que pudieran tener efecto } \\
\text { sobre el resultado: edad, sexo, etc. }\end{array}$ \\
\hline $\begin{array}{l}\text { 3.. Aparte de la intervención en estudio ¿los grupos } \\
\text { fueron tratados en forma similar? } \\
\text { - Si } \\
\text { - No } \\
\text { - No se puede decir }\end{array}$ & \\
\hline
\end{tabular}

C

\section{B.- ¿SON VÁLIDOS LOS RESULTADOS DEL ESTUDIO?} GUIA

COMENTARIOS

\begin{tabular}{|l|l|}
\hline 1. ¿Cuál fue la magnitud del efecto del tratamiento? & $\begin{array}{l}\text { PISTAS: } \\
\text { ¿Qué resultados se midieron? } \\
\text { ¿Qué estimadores se utilizaron? }\end{array}$ \\
\hline $\begin{array}{l}\text { 2... ¿Cuán precisa fue la estimación del efecto del } \\
\text { tratamiento? }\end{array}$ & $\begin{array}{l}\text { PISTA } \\
\text { ¿Cuáles son sus intervalos de confianza? }\end{array}$ \\
\hline
\end{tabular}

C.- ¿ ME AYUDARÁN ESTOS RESULTADOS EN EL CUIDADO DE MIS PACIENTES? GUIA

COMENTARIOS

\begin{tabular}{|c|c|}
\hline $\begin{array}{l}\text { 1. ¿Los resultados pueden ser aplicados en el } \\
\text { cuidado de mis pacientes? } \\
\text { - } \mathrm{Si} \\
\text { - No } \\
\text { - No se puede decir }\end{array}$ & $\begin{array}{l}\text { PISTA } \\
\text { - Los pacientes incluidos en el estudio son } 10 \\
\text { suficientemente parecidos a mis pacientes }\end{array}$ \\
\hline $\begin{array}{l}\text { 2.. ¿Se consideraron todos los resultados de } \\
\text { importancia clinica? } \\
\text { - Si } \\
\text { - No } \\
\text { - No se puede decir }\end{array}$ & $\begin{array}{l}\text { PISTA } \\
\text { - En caso negativo, ¿en qué afecta esto en la decisión } \\
\text { a tomar? }\end{array}$ \\
\hline $\begin{array}{l}\text { 3.. ¿Los beneficios a obtener justifican los riesgos y } \\
\text { los costos del tratamiento? } \\
\text { - } \mathrm{Si} \\
\text { - No } \\
\text { - No se puede decir }\end{array}$ & $\begin{array}{l}\text { PISTA } \\
\text { Es improbable que pueda deducirse del estudio, pero } \\
\text { ¿qué piensa usted al respecto? }\end{array}$ \\
\hline
\end{tabular}

Figs. 5. A, B y C. Guía de usuario para el análisis de artículos de tratamiento y prevención.
La validez interna, se refiere a la proximidad a la verdad. Es un concepto que lleva implícito que la metodología y el diseño empleado garanticen que los resultados no sean sesgados. Es decir, que cuanto más válido es un estudio, mejor se aproxima a la verdad en sus resultados. Para ello, el artículo requiere tener una pregunta o un objetivo claramente definido, contar con procesos de asignación aleatoria y enmascaramiento; que exista similitud de las características basales de los grupos en estudio; que a excepción de la intervención en estudio, los grupos en comparación reciban el mismo tratamiento; que la muestra estudiada tenga un seguimiento adecuado hasta el final del estudio; y, que se haya realizado un análisis por intención de tratamiento.

El impacto del artículo en estudio se relaciona con el tamaño del efecto y no tiene que ver con la significación estadística o el "famoso valor de p" (Manterola $e t$ al., 2008). Se trata en definitiva de valorar por un lado la magnitud del efecto; la que se mide a través del, la razón de probabilidades, el número necesario a tratar, el número necesario a dañar, el cálculo del riesgo relativo y la reducción absoluta de riesgo, etc. (cuando se trata de variables dicotómicas), y a través de la diferencia de medias o de medianas (cuando se trata de variables continuas); y por otro, de determinar la precisión con la que se entregan los resultados; para lo cual se utiliza la determinación de los intervalos de confianza del 95\%, que corresponde al rango de valores (calculado en una muestra) en el cual se encuentra el verdadero valor del parámetro, con una probabilidad determinada, probabilidad que se denomina nivel de confianza.

Finalmente, la validez externa o aplicabilidad, se refiere a la utilidad del artículo analizado en nuestra práctica clínica; o a la reproducibilidad de los resultados en el medio en que se desenvuelve el lector. Es decir, el grado de similitud de los pacientes incluidos, de la intervención realizada, la consideración de todos los resultados clínicamente relevantes en el es- 
tudio, el análisis de los beneficios respecto a perjuicios y costos de la intervención en estudio (incluidos las preferencias del paciente).

Planteado de otro modo, la organización de las guías del usuario se pueden resumir en 3 preguntas ¿Son válidos los resultados del estudio?, ¿Cuáles son los resultados? y ¿Podrán los resultados ayudarme en el cuidado de mis pacientes? Estas, aplican para artículos referentes a tratamiento y prevención (Manterola et al., 2004) (Fig. 5); artículos de relativos a diagnóstico (Fig. 6); a daño, morbilidad o complicaciones (Fig. 7); y pronóstico e historia natural (Fig. 8)
(Evidence-Based Medicine Working Group).

Sin embargo, existen los denominados "artículos integradores"; que no son otra cosa que estudios en los que se trabaja con la evidencia generada a partir de estudios primarios (como los mencionados en el párrafo anterior), la que es en definitiva analizada, integrada y finalmente resumida. En este ámbito, se dispone también de guías de usuario para revisiones sistemáticas de la literatura (Fig. 9), análisis de decisiones (Fig. 10), guías de práctica clínica (Fig. 11), evaluaciones económicas (Fig. 12) y estudios de CVRS (Fig. 13).

\begin{tabular}{|c|c|}
\hline GUIA & COMENTARIOS \\
\hline 1. ¿Son válidos los resultados del estudio? & $\begin{array}{l}\text { Guias primarias } \\
\text { ¿Hubo una comparación independiente y enmascarada con un } \\
\text { patrón de referencia? } \\
\text { ¿La muestra de sujetos incluia un espectro apropiado de } \\
\text { pacientes en los que la prueba diagnóstica será aplicada en la } \\
\text { práctica clínica habitual? } \\
\text { Guías secundarias } \\
\text { ¿Los resultados de la prueba que está siendo evaluada influyeron } \\
\text { en la decisión de realizar el patrón de referencia? } \\
\text { ¿Fueron los métodos para realizar la prueba descritas con } \\
\text { suficiente detalle que permita su replicación? }\end{array}$ \\
\hline 2.¿Cuáles son los resultados? & $\begin{array}{l}\text { ¿Son calculados los likelihood ratios o se presentan los datos } \\
\text { necesarios para su cálculo? }\end{array}$ \\
\hline $\begin{array}{l}\text { 3. ¿Me ayudarán estos resultados en el } \\
\text { cuidado de mis pacientes? }\end{array}$ & $\begin{array}{l}\text { ¿Serán reproducibles los resultados de la prueba y su } \\
\text { interpretación será satisfactoria en mi medio? } \\
\text { ¿Son los resultados aplicables a mis pacientes? } \\
\text { ¿Los resultados cambiarán mi tratamiento? } \\
\text { ¿Cómo resultado de la prueba los pacientes estarán mejor? }\end{array}$ \\
\hline
\end{tabular}

Fig. 6. Guía de usuario para el análisis de artículos de diagnóstico.

GUIA

\begin{tabular}{|l|l|}
\hline 1. ¿Son válidos los resultados del estudio? & $\begin{array}{l}\text { Guias primarias } \\
\text { ¿Fueron claramente identificados los grupos en comparación y } \\
\text { similares con respecto a importantes determinantes de } \\
\text { resultados? } \\
\text { ¿Fueron los resultados y la exposición medidos en la misma } \\
\text { forma en los grupos que están siendo comparados? } \\
\text { ¿Fue el seguimiento lo suficientemente largo y completo? } \\
\text { Guias secundarias } \\
\text { ¿Es la relación temporal correcta? } \\
\text { ¿Hay un gradiente de dosis respuesta? }\end{array}$ \\
\hline $\begin{array}{l}\text { 2. ¿Cuáles son los resultados? } \\
\text { ¿Cuál fuerte es la asociación entre la exposición y el resultado? } \\
\text { ¿Cuán precisa fue la estimación del riesgo? }\end{array}$ \\
\hline cuidado de mis pacientes?
\end{tabular}

Fig. 7. Guía de usuario para el análisis de artículos de etiología y daño o morbilidad. 
GUÍA

COMENTARIOS

\begin{tabular}{|l|l|}
\hline 1. ¿Son válidos los resultados del estudio? & $\begin{array}{l}\text { Guias primarias } \\
\text { ¿Hubo una muestra de pacientes representativa y bien definida } \\
\text { en un punto similar del curso de la enfermedad? } \\
\text { ¿Fue el seguimiento lo suficientemente largo y completo? } \\
\text { Guías secundarias } \\
\text { ¿Fueron usados resultados objetivos y no sesgados? } \\
\text { ¿Hubo ajustes por factores pronósticos relevantes? }\end{array}$ \\
\hline $\begin{array}{l}\text { 2. ¿Cuáles son los resultados? } \\
\text { 3. ¿Me ayudarán estos resultados en } \\
\text { cuidado de mis pacientes? }\end{array}$ & $\begin{array}{l}\text { ¿Cuán grande es el likelihood del evento resultado en un periodo } \\
\text { especifico de tiempo? } \\
\text { ¿Cuán precisa fue la estimación del likelihood? }\end{array}$ \\
\hline $\begin{array}{l}\text { ¿Fueron los pacientes estudiados similares a mis propios } \\
\text { pacientes? } \\
\text { ¿Los resultados conducirán directamente a seleccionar o evitar } \\
\text { la terapia? } \\
\text { ¿Son los resultados útiles para aconsejar a los pacientes? }\end{array}$ \\
\hline
\end{tabular}

Fig. 8. Guía de usuario para el análisis de artículos de pronóstico e historia natural.

\begin{tabular}{|c|c|}
\hline GUIA & COMENTARIOS \\
\hline 1. ¿Son válidos los resultados del estudio? & $\begin{array}{l}\text { Guias primarias } \\
\text { ¿La revisión está focalizada en una pregunta clínica? } \\
\text { ¿Fueron los criterios utilizados para seleccionar los articulos } \\
\text { incluidos apropiados? } \\
\text { Guías secundarias } \\
\text { ¿Es poco probable que sea relevante, pero estudios } \\
\text { relevantes fueron perdidos? } \\
\text { ¿Fue evaluada la validez de los estudios incluidos? } \\
\text { ¿Fue evaluada la reproducibilidad de los estudios? } \\
\text { ¿Los resultados fueron similares entre los estudios? }\end{array}$ \\
\hline 2. ¿Cuáles son los resultados? & $\begin{array}{l}\text { ¿Cuál es el resultado global de la revisión? } \\
\text { ¿Cuán precisos son los resultados? }\end{array}$ \\
\hline $\begin{array}{l}\text { 3. ¿Me ayudarán estos resultados en el } \\
\text { cuidado de mis pacientes? }\end{array}$ & $\begin{array}{l}\text { ¿Pueden los resultados ser aplicados al cuidado de mis } \\
\text { pacientes? } \\
\text { ¿Fueron todos los resultados clinicamente importantes } \\
\text { considerados? } \\
\text { ¿Los beneficios justifican los riesgos y costos? }\end{array}$ \\
\hline
\end{tabular}

Fig. 9. Guía de usuario para el análisis de revisiones sistemáticas de la literatura.

GUÍA

\begin{tabular}{|l|l|}
\hline 1. ¿Son válidos los resultados del estudio? & $\begin{array}{l}\text { ¿Fueron todas las estrategias y resultados importantes incluidos? } \\
\text { ¿Hubo un proceso explicito y sensible utilizado para identificar, } \\
\text { seleccionar y combinar la evidencia en probabilidades? } \\
\text { ¿Fueron las utilidades obtenidas de una manera explicita y } \\
\text { sensible desde fuentes creibles? } \\
\text { ¿Fue considerado el potencial impacto de cualquier incerteza en } \\
\text { la evidencia determinada? }\end{array}$ \\
\hline $\begin{array}{l}\text { 2. ¿Cuáles son los resultados? } \\
\text { 3. ¿Me ayudarán estos resultados en el } \\
\text { cuidado de mis pacientes? }\end{array}$ & $\begin{array}{l}\text { ¿En el análisis basal, alguna estrategia resultó en una importante } \\
\text { ganancia clinica para los pacientes? Si no ¿es el resultado un } \\
\text { toss-up? } \\
\text { ¿Cuán fuerte es la evidencia usada en el análisis? } \\
\text { ¿Podria la incerteza en la evidencia cambiar el resultado? }\end{array}$ \\
\hline $\begin{array}{l}\text { ¿La probabilidad estimada fit las caracteristicas clínicas de mis } \\
\text { ¿La utilidad refleja cómo mis pacientes podrian valorar los } \\
\text { resultados de la decisión? }\end{array}$ \\
\hline
\end{tabular}

Fig. 10. Guía de usuario para el análisis de artículos de análisis de decisiones. 
MANTEROLA, C. \& OTZEN, T. Análisis crítico de la literatura biomédica. Int. J. Morphol., 32(2):599-607, 2014.

GUIA

\begin{tabular}{|l|l|}
\hline 1. ¿Son válidos los resultados del estudio? & $\begin{array}{l}\text { Guias primarias } \\
\text { ¿Fueron todas las opciones y resultados claramente } \\
\text { especificados? } \\
\text { ¿El proceso usado para identificar, seleccionar y combinar } \\
\text { evidencia fue explicito y sensible? } \\
\text { Guias secundarias } \\
\text { ¿Hubo un proceso explicito y sensible usado para considerar el } \\
\text { valor relativo de los diferentes resultados? } \\
\text { ¿Es probable que la guia de cuenta de importante desarrollo } \\
\text { reciente? } \\
\text { ¿La guia ha sido sometida a revisiones y pruebas? }\end{array}$ \\
\hline 2. ¿Cuáles son los resultados? & $\begin{array}{l}\text { ¿Son hechas recomendaciones importantes prácticas y clínicas? } \\
\text { ¿Cuán fuerte son las recomendaciones? } \\
\text { ¿Cuál es el impacto de la incerteza asociada con la evidencia y } \\
\text { los valores usados en la guia? }\end{array}$ \\
\hline $\begin{array}{l}\text { 3. ¿Me ayudarán estos resultados en el } \\
\text { cuidado de mis pacientes? }\end{array}$ & $\begin{array}{l}\text { ¿Es el objetivo primario de la guia consistente con su objetivo? } \\
\text { ¿Son las recomendaciones aplicables a sus pacientes? }\end{array}$ \\
\hline
\end{tabular}

Fig. 11. Guía de usuario para el análisis de guías de práctica clínica.

\section{COMENTARIOS}

\begin{tabular}{|l|l|}
\hline 1. ¿Son válidos los resultados del estudio? & $\begin{array}{l}\text { ¿El análisis proporciona una completa comparación económica } \\
\text { de las estrategias de atención de salud? } \\
\text { ¿Fueron los costos y resultados adecuadamente medidos y } \\
\text { valorados? } \\
\text { ¿Fue apropiada la asignación para las incertezas en el análisis? } \\
\text { ¿Son estimados los costos y los resultados relacionados con el } \\
\text { riesgo basal en la población en tratamiento? }\end{array}$ \\
\hline $\begin{array}{l}\text { 2. ¿Cuáles son los resultados? } \\
\text { 3. ¿Me ayudarán estos resultados en el } \\
\text { cuidado de mis pacientes? }\end{array}$ & $\begin{array}{l}\text { ¿Cuáles fueron los costos incrementales y los resultados de cada } \\
\text { estrategia? } \\
\text { ¿Los costos incrementales y los resultados difieren entre } \\
\text { subgrupos? } \\
\text { ¿Cuánto cambia los resultados la asignación de las incertezas? }\end{array}$ \\
\hline $\begin{array}{l}\text { ¿Son los beneficios del tratamiento peores mejores que los } \\
\text { ¿iesgos y costos? } \\
\text { ¿Podria esperar costos similares? }\end{array}$ \\
\hline
\end{tabular}

Fig. 12. Guía de usuario para el análisis de artículos de análisis económicos.

GUIA

COMENTARIOS

\begin{tabular}{|l|l|}
\hline 1. ¿Son válidos los resultados del estudio? & $\begin{array}{l}\text { Guias primarias } \\
\text { ¿Han los investigadores medido aspectos de la vida de los } \\
\text { pacientes que los pacientes consideran importantes? } \\
\text { ¿El instrumento de medición de CVRS trabaja en la forma en que } \\
\text { se supone que lo haga? } \\
\text { Guias secundarias } \\
\text { ¿Hay aspectos importantes de CVRS que han sido omitidos? } \\
\text { ¿Si hay trade-offs entre calidad y cantidad de vida, o una } \\
\text { evaluación económica, han usado las mediciones adecuadas? }\end{array}$ \\
\hline $\begin{array}{l}\text { 2. ¿Cuáles son los resultados? } \\
\begin{array}{l}\text { 3. ¿Me ayudarán estos resultados en el } \\
\text { cuidado de mis pacientes? }\end{array}\end{array}$ & $\begin{array}{l}\text { ¿La información del estudio me ayudará a informar a mis } \\
\text { pacientes? } \\
\text { ¿El diseño del estudio simula la práctica clínica? }\end{array}$ \\
\hline
\end{tabular}

Fig. 13. Guía de usuario para el análisis de artículos de CVRS. 


\section{Aplicación práctica}

La experiencia nos dice que el volumen de publicaciones es cuantioso, y se estima que las aquellas referentes a tratamiento corresponden a un $35 \%$ a $45 \%$ del total. Por otra parte, la publicación de un artículo incluso en revistas prestigiosas, no es garantía de la calidad de lo informado (Manterola et al., 2004, 2006a, Pineda et al., 2005).

Es por esto que, se han desarrollado estrategias para realizar un tamizado rápido y descartar aquellos artículos que no ameritan ser leídos, de forma tal de optimizar el poco tiempo disponible para lectura. La primera selección, ya sea a partir del resumen de una revista o del resultado de una búsqueda bibliográfica, se hace en función del título, buscando especialmente artículos originales del mejor nivel de evidencia posible; es decir que si nuestra inquietud es relacionada con tratamiento, una respuesta apropiada podríamos encontrarla en estudios del tipo ensayos clínicos individuales o multicéntricos con asignación aleatoria, doble o triple enmascaramiento e intervalo de confianza pequeño; y revisiones sistemáticas de la literatura con metaanálisis.

A veces, puede ser de ayuda prestar atención a los autores (cual es su trayectoria en el tema), identificar el lugar en el que se ha realizado el trabajo (permite valorar si los resultados obtenidos pudiesen ser reproducidos en mi realidad). Posteriormente, para realizar una primera valoración a menudo es suficiente con la lectura del resumen, evaluando su (s) objetivo (s), si la información que contiene sobre el diseño del estudio permite suponer que es metodológicamente adecuado y si, en el supuesto de que la conclusión del estudio sea válida, tiene relevancia clínica y es aplicable a la situación concreta que se desea resolver. Esto permitirá descartar un número muy significativo de artículos, hecho que no significa que nos quedaremos con los resultados y conclusiones de aquellos que nos parecieron interesantes. Por el contrario, estos serán los que analizaremos con mayor detención.

Las guías del usuario contienen elementos que permiten objetivar la calidad de los resultados y si éstos pueden o no ser aplicados en el cuidado de nuestros pacientes. Está compuesta por 2 columnas, a la izquierda la guía propiamente tal, y a la derecha una serie de "pistas" o acotaciones que ayudan a responder en forma más apropiada la guía. En las próximas líneas se aplicará este instrumento en forma práctica a un artículo de tratamiento publicado en una prestigiosa revista científica que dice relación los resultados obtenidos con el uso de cirugía y quimioradioterapia vs. cirugía exclusiva en pacientes con adenocarcinoma gástrico y de la unión esofagogástrica (Macdonald et al., 2001). El protocolo publicado en este artículo ha sido incorporado en los hospitales chilenos como tratamiento del cáncer gástrico a pesar que la metodología y los resultados del estudio son discutibles, lo que hace objetable la validez interna y externa de los resultados (Manterola et al., 2006b). En negritas y subrayado se encuentra la respuesta a la pregunta de la guía.

\section{A.- ¿Son válidos los resultados del estudio?}

Preguntas de "eliminación": si el artículo no responde adecuadamente las siguientes tres preguntas, no es aconsejable continuar con la lectura crítica (Tabla I).

Tabla I. ¿Son válidos los resultados del estudio?

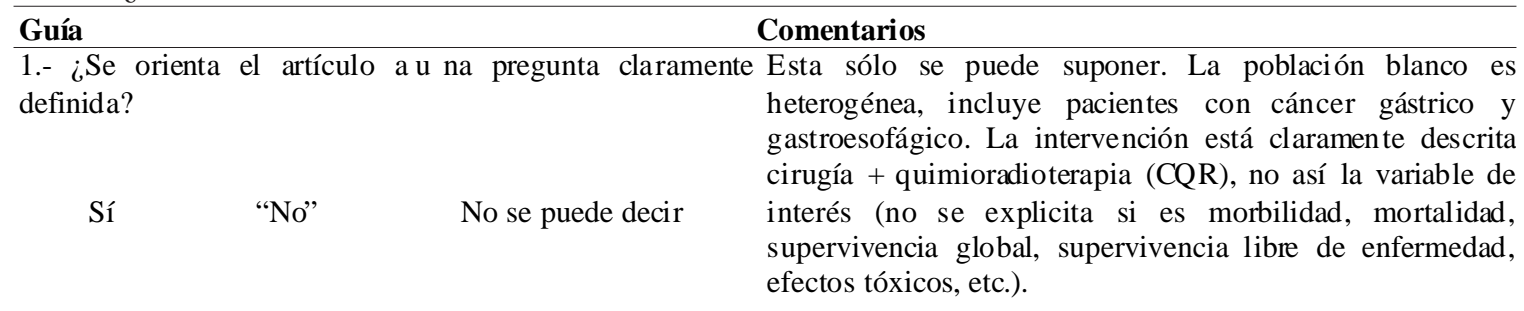

2.- ¿Fue aleatoria la asignación de los pacientes al No se describe que método de asignación fue utilizado, tratamiento? sólo se especifica que 281 pacientes fueron asignados a CQR y 275 a cirugía exclusiva (CE), pero en el postoperatorio. No se mantuvo oculta la secuencia de asignación.

3.- ¿Fueron adecuadamente considerados hasta el final del Los pacientes fueron analizados en los grupos a los que estudio todos los pacientes que entraron a él? fueron asignados, pero el seguimiento sólo se completó en el grupo de CE $(35,6 \%$ de los pacientes del grupo de CQR Sí $\quad$ No No se puede decir" no completó tratamiento). 
Tabla II. Preguntas de detalle.

Guía Comentarios

1.- ¿Estuvieron los pacientes, el personal de salud y el p ersonalLos pacientes ye l personal que aplicó las terapias no se involucrado en el estudio enmascarados ("ciegos") respecto delencontraban enmascarados. No se especificó si los observadores tratamiento?

Sí "No" No se puede decir

2.- Los grupos en estudio ¿eran similares al inicio del estudio?

Sí No "No se puede decir" estuvieron enmascarados, pero el tatuaje previo a la radioterapia es evidente a la simple inspección del abdomen. Por ende, es improbable un enmascaramiento de los observadores.

Al observar la Tabla 1 del artículo original pareciera que sí, pero existen una serie de factores que pueden tener efecto sobre los resultados y que no fueron considerados (tipo histológico, grado de diferenciación, existencia de enfermedades coexistentes, duración síntomas, estado inmunológico y nutricional, necesidad de transfusiones), por lo que es difícil establecer si los grupos eran absolutamente similares.

3.- Aparte de la intervención en estudio ¿los grupos fueronHay incertidumbre en relación al tipo de resección y l a tratados en forma similar?

linfadenectomía realizada. Hay muchos casos de D0, no queda clara la proporción de pacientes D0, D1 y D2 por grupos (se menciona que sólo un $10 \%$ de los pacientes fueron resecados con D2 y un $36 \%$ con D1); y, por otra parte, se verifica que el $35 \%$ de Sí "No" No se puede decir los pacientes sufrió alguna modificación de su esquema terapéutico original y un $6,5 \%$ fue desviado a otro protocolo, del que no se menciona información adicional.

Tabla III. ¿Cuáles fueron los resultados?

1.- ¿Cuál fue la magnitud del efecto del tratamiento?

2. ¿Cuán precisa fue la estimación del efecto del tratamiento?

\section{Comentarios}

Se evaluaron mediana de supervivencia global, supervivencia real a 36 meses, supervivencia global libre de enfermedad, supervivencia real libre de enfermedad a 36 meses y recurrencia: resultados que se reportan en la Tabla 4 del estudio original.

La precisión de la estimación del efecto, fue realizada mediante el cálculo de los hazard ratios con sus respectivos intervalos $\mathrm{d} \epsilon$ confianza de $95 \%$, datos que se de scriben en la Tabla 4 del estudic original.

Tabla IV. ¿Me ayudarán los resultados en el cuidado de mis pacientes?

\section{Guía}

\section{Comentarios}

pacientes?

$$
\text { Sí “No" No se puede decir }
$$

2.- ¿Se consideraron todos los resultados de importancia clínica?

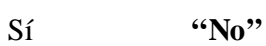

3.- ¿Los beneficios a obtener justifican los riesgos y los costos delNo se reporta información sobre complicaciones quirúrgicas; por tratamiento?

Sí "No" diferencias biodemográficas respecto de nuestra realidad (la población de raza negra, asiática y a nglosajona en nuestra realidad es escasa; y e 1 grupo de denominado "otros" que representa la población latina es sólo el $4 \%$ de la muestra); más del $50 \%$ de las lesiones son antrales, y en nuestro medio un porcentaje mayoritario son de fondo gástrico; al mismo tiempo, en nuestra realidad existe una baja prevalencia de lesiones T1 y $\mathrm{T} 2$ (mayoritaria en el artículo en evaluación).

Variables como: calidad de vida, efectos adversos de la terapia en evaluación, mortalidad por efectos deletéreos, morbilidad y mortalidad quirúrgica y costos involucrados no fueron consideradas (al menos no aparecen consignadas en el reporte del estudio). otra parte, un $36 \%$ de $\mathrm{p}$ acientes no completaron el esquema asignado, por lo que las estimaciones finales se realizan en base al 64\% de los pacientes del grupo de CQR; lo que difículta la valoración de potenciales beneficios del esquema $\mathrm{CQR}$ al compararlo con CE. 
Hasta esta etapa, por no existir elementos que permitan dar respuesta a las preguntas de eliminación, se considera que el artículo no las supera; razón por la cual no valdría la pena seguir adelante con su lectura y análisis. Sin embargo, como se trata de desarrollar un ejemplo, continuaré con el desarrollo de la guía de usuario. Tabla II.

B.- ¿Cuáles fueron los resultados? (Tabla III).

C.- ¿Me ayudarán los resultados en el cuidado de mis pacientes? (Tabla IV).
En resumen, se trata de un ensayo clínico multicéntrico, sin asignación aleatoria precisa, ni enmascaramiento, con una estimación errónea del tamaño de la muestra, en el que se mezclan poblaciones con diversos tipos de cánceres; cuya validez interna se encuentra afectada por lo que su nivel de evidencia es $2 \mathrm{~b}$. Por otra parte, en relación a la validez externa; si se omitiesen los problemas relacionados con la validez interna, se constata que la inferencia de los resultados sólo aplicaría a poblaciones de características similares a las del estudio. En este caso, no a la población chilena.

MANTEROLA, C. \& OTZEN, T. Critical analysis of biomedical literature. Int. J. Morphol., 32(2):599-607, 2014.

SUMMARY: Given the large number of publications, it is a problem to choose a study to respond to clinical problems with a good level of evidence. This requires following a scientific methodology, defining and limiting the problem, transforming it into a question, applying a search strategy in an appropriate source of information and finally, analyzing the information and summarizing the evidence found. Specific tools for analize the evidence had been develop. They are known as reading guides, Reader's Guides or Users' Guides, of which there are distinct formats, but in general, they give the necessary guidelines for the critical analysis of the different tipes of biomedical articles. These tools assess three key areas of study: internal validity, impact and external validity. The areas above mentioned are practically evaluated applying a user's guide to articles relating to treatment, as an example to a study published in a prestigious scientific journal.

KEY WORDS: Criticall appraisal of biomedical articles; Evidence-Based practice; Reader's guides; Users' guides.

\section{REFERENCIAS BIBLIOGRÁFICAS}

Evidence-Based Medicine Working Group. Evidence-based medicine. A new approach to teaching the practice of medicine. JAMA, 268:2420-25, 1992.

Macdonald, J. S.; Smalley, S. R.; Benedetti, J.; Hundahl, S. A.; Estes, N. C.; Stemmermann, G. N.; Haller, D. G.; Ajani, J. A.; Gunderson, L. L.; Jessup, J. M. \& Martenson, J. A. Chemoradiotherapy after surgery compared with surgery alone for adenocarcinoma of the stomach or the gastroesophageal junction. N. Engl. J. Med., 345(10):725-30, 2001.

Manterola C. El proceso que conduce al desarrollo de la investigación científica. Su aplicación en cirugía. Rev. Chil. Cir., 53:1049, 2001.

Manterola, C.; Vial, M. \& Pineda, V. Revisión crítica de la literatura para artículos de terapia. Rev. Chil. Cir., 56:604-9, 2004.

Manterola, C.; Pineda, V.; Vial, M.; Losada, H. \& MINCIR Group. What is the methodologic quality of human therapy studies in ISI surgical publications? Ann. Surg., 244(5):827-32, 2006 a.

Manterola, C.; Torres-Quevedo, R.; Burgos, L.; Pineda, V. \& Vial, M. Methodological quality of an article on the treatment of gastric cancer adopted as protocol by some Chilean hospitals. Rev. Med. Chile, 134:920-6, 2006 b.

Manterola, C. \& Pineda, V. \& Grupo MINCIR. El valor de "p" y la "significación estadística". Aspectos generales y su valor en la práctica clínica. Rev. Chil. Cir., 60:86-9, 2008.
Manterola C. Medicina Basada en la Evidencia o Medicina Basada en Pruebas. Generalidades acerca de su aplicación en la práctica clínica cotidiana. Rev. Med. Clin. Condes, 20:125-30, 2009.

Pineda, V.; Manterola, C.; Vial, M. \& Losada, H. ¿Cuál es la calidad metodológica de los artículos referentes a terapia, publicados en la revista chilena de cirugía? Rev. Chil. Cir., 57:5007, 2005.

\section{Dirección para Correspondencia: \\ Dr. Carlos Manterola \\ Departamento de Cirugía \\ Universidad de La Frontera \\ Casilla 54-D, Temuco \\ CHILE}

Email: carlos.manterola@ufrontera.cl

Recibido : 11-01-2014

Aceptado: 22-02-2014 